Portland State University

PDXScholar

$11-1-1988$

\title{
Roughness-induced resonance for molecular fluorescence near a corrugated metallic surface
}

\author{
P.T. Leung \\ Portland State University \\ Young Sik Kim \\ Thomas F. George
}

Follow this and additional works at: https://pdxscholar.library.pdx.edu/phy_fac

Part of the Physics Commons

Let us know how access to this document benefits you.

\section{Citation Details}

Leung, P. T., Young Sik, K., \& George, T. F. (1988). Roughness-induced resonance for molecular fluorescence near a corrugated metallic surface. Physical Review B (Condensed Matter), 38(14), $10032-10034$.

This Article is brought to you for free and open access. It has been accepted for inclusion in Physics Faculty Publications and Presentations by an authorized administrator of PDXScholar. Please contact us if we can make this document more accessible: pdxscholar@pdx.edu. 


\title{
Brief Reports
}

Brief Reports are short papers which report on completed research which, while meeting the usual Physical Review standards of scientific quality, does not warrant a regular article. (Addenda to papers previously published in the Physical Review by the same authors are included in Brief Reports.) $A$ Brief Report may be no longer than $3 \frac{1}{2}$ printed pages and must be accompanied by an abstract. The same publication schedule as for regular articles is followed, and page proofs are sent to authors.

\section{Roughness-induced resonance for molecular fluorescence near a corrugated metallic surface}

\author{
P. T. Leung, ${ }^{*}$ Young Sik Kim, ${ }^{\dagger}$ and Thomas F. George \\ Department of Chemistry and Department of Physics and Astronomy, State University of New York at Buffalo, \\ 239 Fronczak Hall, Buffalo, New York 14260
}

(Received 14 March 1988)

\begin{abstract}
Based on a dynamical energy-transfer theory for the molecular decay rate near a rough metallic surface, a new resonance structure is predicted which is mediated by the roughness and which is absent in the static image theory. The implication of this new resonance structure to other phenomena, such as electron-energy-loss spectrum and cross coupling in thin films, is pointed out.
\end{abstract}

In two previous publications, ${ }^{1,2}$ we have considered the problem of the decay rates for molecules in the vicinity of a periodically roughened (sinusoidal grating) metallic surface in the context of both the static image theory ${ }^{1}$ (IT) and the more accurate dynamical energy-transfer theory $(E T){ }^{2}$ The same problem for the case of a randomly roughened surface has also been treated some time earlier in the context of IT. ${ }^{3}$ The motivation of all this work comes from recent fluorescence experiments ${ }^{4}$ in which discrepancies were found when compared to the wellestablished theory for flat surfaces. ${ }^{5}$ In Ref. 2 , we pointed out that the simpler theory (IT) can be very inaccurate for highly conducting substrates or large moleculesurface distances $(d)$, and we have further established a better theory (ET) for rough surfaces in a perturbative approach. In this Brief Report, we want to point out that, aside from giving the more accurate results for large $d$, this dynamical theory ${ }^{2}$ contains a novel resonance structure (in addition to the well-known surface plasmon for flat surface, i.e., $\omega_{\mathrm{sp}} \simeq \omega_{P}^{\text {bulk }} / \sqrt{2}$ ) which is induced by the presence of the surface roughness and which is absent from the cruder theory (IT). ${ }^{1,3}$ The implications to earlier electron-scattering experiments and the relation to recent fluorescence experiments on a corrugated thin film are discussed.

Let us start by recalling some previous results for a molecular dipole located at a distance $d$ from a sinusoidal grating of amplitude $\zeta_{0}$ and period $Q$ and oriented perpendicular to the grating characterized by a complex dielectric function $\epsilon(\omega)$, where $\omega$ refers to the emission frequency of the dipole. According to IT, the normalized decay rate (with respect to the free-molecule value) for this case is obtained as ${ }^{1}$

$$
\frac{\gamma_{\mathrm{IT}}}{\gamma_{0}}=1+\frac{3}{2 k^{3}} \operatorname{Im} G_{\mathrm{IT}},
$$

where $k$ is the emission wave number and $G_{\mathrm{IT}}$ is given by

$$
\begin{aligned}
G_{\mathrm{IT}}(\omega)= & \frac{1}{4 d^{3}} \frac{\epsilon-1}{\epsilon+1} \\
& +\frac{4 \zeta_{0}}{\pi} \frac{\epsilon-1}{(\epsilon+1)^{2}} \int_{0}^{\infty} d u \int_{0}^{\infty} d v(\epsilon f g+h) e^{-(f+g) d}
\end{aligned}
$$

with the functions $f, g$, and $h$ given by

$$
\begin{aligned}
& f(u, v)=\left[(u+Q / 2)^{2}+v^{2}\right]^{1 / 2}, \\
& g(u, v)=\left[(u-Q / 2)^{2}+v^{2}\right]^{1 / 2}, \\
& h(u, v)=u^{2}+v^{2}-Q^{2} / 4 .
\end{aligned}
$$

On the other hand, the corresponding ratio $\gamma_{\mathrm{ET}} / \gamma_{0}$ has been obtained in a form similar to that in Eq. (1), with $G_{\mathrm{ET}}$ given by ${ }^{2}$

$$
\begin{aligned}
G_{\mathrm{ET}}(\omega)= & -k^{3} \int_{0}^{\infty} d u R \frac{u^{3}}{l_{1}} e^{-2 l_{1} \bar{d}} \\
& -i k^{3} \zeta_{0} \frac{\epsilon-1}{k_{1}-\epsilon k_{2}} Q^{2} e^{i k_{2} d} \\
& \times \int_{0}^{\infty} d u(1-R) \frac{u^{3}}{l_{1}} e^{-l_{1} \bar{d}},
\end{aligned}
$$

where $\quad R=\left(l_{2}-\epsilon l_{1}\right) /\left(l_{2}+\epsilon l_{1}\right), \quad l_{1}=-i\left(1-u^{2}\right)^{1 / 2}$, $l_{2}=-i\left(\epsilon-u^{2}\right)^{1 / 2}, \bar{d}=k d$, and $k_{1}$ and $k_{2}$ are defined as

$$
\begin{aligned}
& k_{1}=-\left(\epsilon k^{2}-Q^{2}\right)^{1 / 2}, \\
& k_{2}= \begin{cases}\left(k^{2}-Q^{2}\right)^{1 / 2}, & k^{2}>Q^{2} \\
i\left(Q^{2}-k^{2}\right)^{1 / 2}, & k^{2}<Q^{2} .\end{cases}
\end{aligned}
$$

It is interesting to point out that in spite of the very different mathematical forms as they appear in Eqs. (2) and (4), it has been observed numerically that a comparison between the two for different ranges of $d$ indeed shows the correct trend that one would expect to be exhibited between a dynamical and a static (nonretarded) 
theory. ${ }^{2}$ Here we want to point out that there is an extra resonance feature which is predicted by Eq. (4) and not by Eq. (2). This feature was overlooked in the previous paper $^{2}$ and should have some relevance to certain recent experiments.

It is not difficult to see from Eq. (2) that the resonance structure implied by IT arises from the condition $\epsilon(\omega)+1=0$, which in turn leads to the well-known flat surface-plasmon resonance frequency $\omega_{\mathrm{sp}}=\omega_{p}^{\mathrm{bulk}} / \sqrt{2}$. Hence one is led to the conclusion that the surface roughness will not shift the peak in the decay rate spectrum within IT, and this has been observed from previous calculations. ${ }^{1,3}$ However, from Eq. (4) one observes two resonance factors-one from $R$ and the other from

$$
S=k_{1}-\epsilon k_{2} \text {. }
$$

It is well known ${ }^{5}$ that the peak implied from $R$ (which already occurs in the case of a flat surface) is essentially the same as that in IT which occurs at $\omega \simeq \omega_{\text {sp }}$, but the one implied from $S$ is unique in ET and depends on the value of $Q$. For $k<Q$, one can easily show from Eqs. (5) and (6) that

$$
S \sim i Q(\epsilon+1),
$$

which means that the peaks implied from $R$ and $S$ will overlap and be at the same position as that in the flat surface case. For the other extreme where $k \gg Q, S$ reduces to

$$
S \sim-\sqrt{\epsilon} k(\sqrt{\epsilon}+1) .
$$

This would imply poles very close to the previous case if we consider noble metals like silver, which have a very small value for the imaginary part of their dielectric functions. However, for $Q \sim k$, Eq. (6) will induce another resonance peak in the decay-rate spectrum, as we shall see below.

In Fig. 1, we show the decay-rate spectrum for different roughness parameters $\left(\xi_{0}, Q\right)$ with $d$ fixed at 50 $\AA$ for a $\mathrm{Ag}$ grating. At such a close distance, the two theories (ET and IT) give results very close to each other ${ }^{2}$ except in Figs. 1(b) and 1(c) where $Q \sim k$; here we observe an extra peak induced by $Q$ whose position depends on the magnitude $Q$ and whose magnitude depends on $\zeta_{0}$. It is worth noting that this new peak grows as $\zeta_{0}$ increases and may actually overshadow the $\omega_{\text {sp }}$ peak (at $\sim 3.6 \mathrm{eV}$ for $\mathrm{Ag}$ ) for large enough values of $\zeta_{0}$ (although we cannot show a numerical example here since such a case will exceed the limit of our present first-order perturbation theory). When this happens, it is possible that it would appear in actual experiments as if the $\omega_{\text {sp }}$ peak is "shifted" due to the presence of the surface roughness.

It is interesting to mention that in a previous analysis, Rahman and Maradudin ${ }^{6}$ (RM) applied the imagepotential theory established by them for rough surfaces to analyze the results reported in an experiment of electron scattering on a Mo surface carried out some time earlier by Lecante, Ballu, and Newns (LBN), ${ }^{7}$ in which a shift in the peak frequency is observed in the energy-loss spectrum. According to the RM analysis, the shift in the LBN experiment cannot be explained by surface rough-

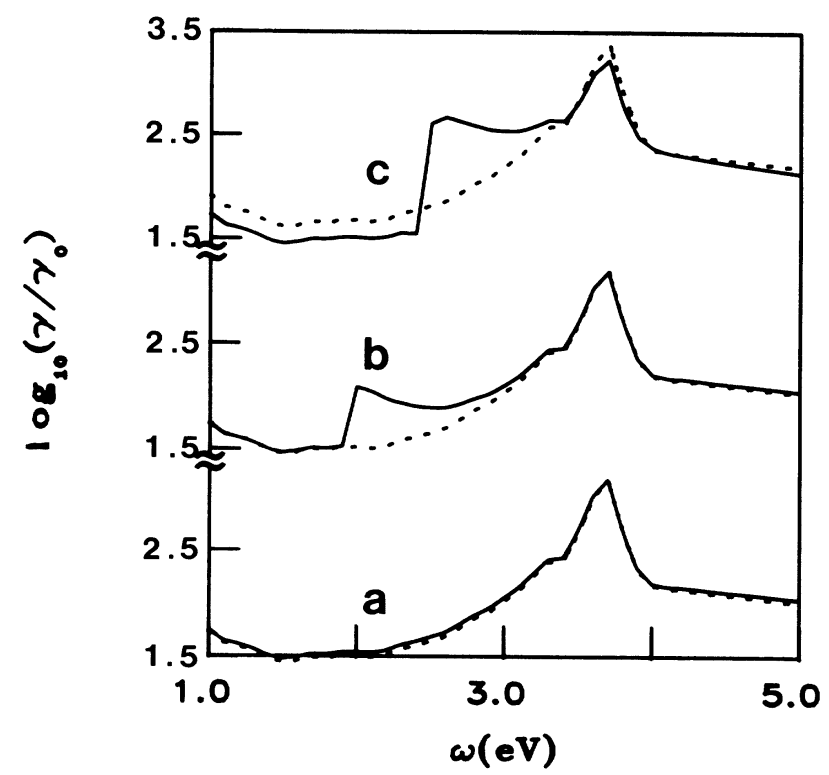

FIG. 1. Decay-rate spectrum for a molecule located at $d=50$ $\AA$ above a sinusoidal $\mathrm{Ag}$ grating of varying roughness parameters $\left(\zeta_{0}, Q\right)$. Curve $a, \zeta_{0}=1 \AA, Q=1 \times 10^{-2} \AA-1$; curve $b, \zeta_{0}=1$ $\AA, Q=1 \times 10^{-3} \AA^{-1}$; curve $c, \zeta_{0}=10 \AA, Q=1.25 \times 10^{-3} \AA^{-1}$. The solid line represents the results from the dynamical theory (ET) and the dotted line the results from the image theory (IT).

ness as LBN have claimed. However, as in our present analysis of the fluorescence spectrum for the admolecules, such a roughness-induced (or "shifted") peak can only occur in the dynamical theory but not in IT. Hence it is possible that the original speculation by LBN may still be correct if retardation effects are taken into account in the RM analysis.

Another situation where this roughness-induced resonance may play a role is in the recent fluorescence light scattering experiment carried out by Gruhlke, Holland, and $\mathrm{Hall}^{8}$ on a corrugated thin silver film in which the effect of surface-plasmon cross coupling is clearly observed. We believe that the roughness-induced resonance effect pointed out here is a necessary condition for the cross-coupling phenomenon to occur. It would be interesting to generalize the result in Eq. (4) to the corrugated film system and look for such a cross-coupling phenomenon.

In summary, we stress that this roughness-induced resonance effect should be general and should occur in phenomena other than fluorescence and for rough surfaces other than a grating, such as one with randomly (Gaussian) distributed roughness. ${ }^{3,6}$ The reasons that the previous analyses ${ }^{1-3,6}$ did not yield such peak structure are due simply to the fact that either the nonretarded theory (IT) has been applied or the values of $Q$ are too extreme to lie too far away from the emission wave numbers.

This research was supported by the Air Force Office of Scientific Research, Air Force Systems Command (AFSC), United States Air Force, under Contract No. F49620-86-C-0009, by the U. S. Office of Naval Research, and by the National Science Foundation under Grant No. CHE-86-20274. 
*Present address: Department of Physics, Portland State Uni+ versity, Portland, OR 97201.

Also at Department of Chemistry, University of Rochester, Rochester, NY 14627.

${ }^{1}$ P. T. Leung, Z. C. Wu, D. A. Jelski, and T. F. George, Phys. Rev. B 36, 1475 (1987).

${ }^{2}$ P. T. Leung and T. F. George, Phys. Rev. B 36, 4664 (1987).

${ }^{3}$ J. Arias, P. K. Aravind, and H. Metiu, Chem. Phys. Lett. 85, 404 (1982).

${ }^{4}$ R. Rossetti and L. E. Brus, J. Chem. Phys. 76, 1146 (1982); A.
P. Alivisatos, D. H. Waldeck, and C. B. Harris, ibid. 82, 541 (1985).

${ }^{5}$ R. R. Chance, A. Prock, and R. Silbey, Adv. Chem. Phys. 37, 1 (1978).

${ }^{6}$ T. Rahman and A. A. Maradudin, Phys. Rev. B 21, 504 (1980).

${ }^{7}$ J. Lecante, Y. Ballu, and D. M. Newns, Phys. Rev. Lett. 38, 36 (1977).

${ }^{8}$ R. W. Gruhkle, W. R. Holland, and D. G. Hall, Phys. Rev. Lett. 56, 2838 (1986). 